
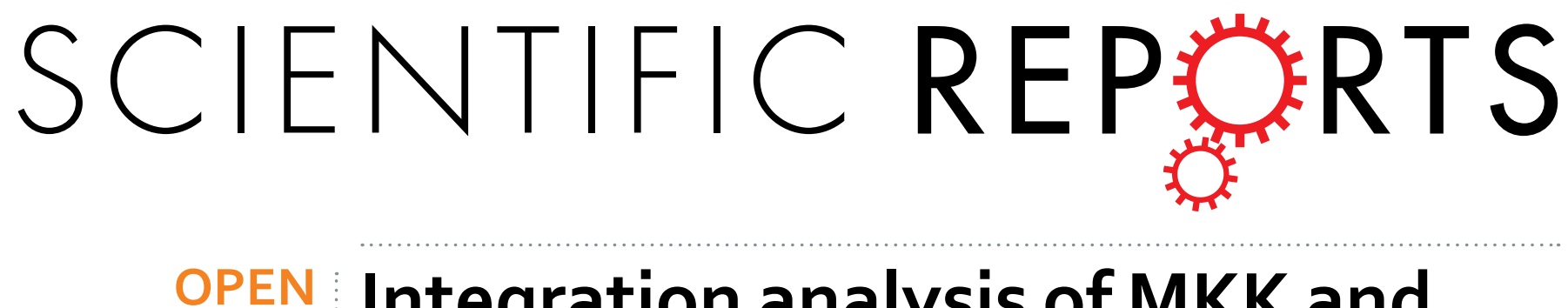

\title{
Integration analysis of MKK and MAPK family members highlights potential MAPK signaling modules in cotton
}

Mitogen-activated protein kinase (MAPK) cascades play a crucial role in plant growth and development, as well as their biotic and abiotic stress responses. As a nodal point of the MAPK cascade, the MKK gene family has not been systematically studied in cotton. Here, we identified 11 putative MKK genes in the Gossypium raimondii genome. Phylogenetic analysis showed that the MKKs were supported by architectures of conserved protein motifs. Expression patterns of MKKs under hormone treatments or abiotic stresses revealed their diverse functions in stress responses. Based on a yeast two hybrid, a total of 63 interactive pairs of MKKs and MAPKs were identified in cotton. Among these, 40 interactive pairs were newly identified compared to that reported previously in Arabidopsis. Integration analysis of the interaction network and expression patterns of MKK and MAPK family members revealed 13 potential MAPK signaling modules that are involved in the complicated cross-talk between hormones and abiotic stresses. Taken together, our data enhance the understanding of the evolution and function of MAPK cascades in cotton, and lay the foundation for the improvement of various defense responses that use MAPK signaling modules in the future.

Mitogen-activated protein kinase (MAPK) cascades are evolutionarily conserved and fundamental signal transduction pathways that play roles upstream of various receptors/sensors that transduce extracellular stimuli into intracellular responses in eukaryotes. A canonical MAPK cascade consists of three functionally linked protein kinases. MAPKs are phosphorylated at their threonine and tyrosine (TXY) residues and are activated by a MAPK kinase (MAPKK), which itself is phosphorylated at its serine/threonine residues in the S/T motif and is activated by MAPKK kinase (MAPKKK). Numerous reports have provided evidence that the MAPK cascades play pivotal roles in the transduction of diverse cellular processes, such as salinity stress, drought, temperature, wounding, pathogen attack and plant hormone responses ${ }^{1-3}$.

Many members of MAPK cascades have been identified in a variety of plants after the completion of the whole genome sequence. A total of 80 MAPKKKs, 10 MAPKKs and 20 MAPKs have been characterized in the Arabidopsis genome ${ }^{4,5} ; 75$ MAPKKKs, 8 MAPKKs and 17 MAPKs are present in the O. sativa genome ${ }^{6,7} ; 89$ MAPKKKs, 6 MAPKKs and 16 MAPKs have been reported in tomato ${ }^{8,9} ; 74$ putative MAPKKKs, 9 MAPKKs and 19 MAPKs have been found in maize ${ }^{10,11}$; 59 MAPKKKs, 6 MAPKKs and 14 MAPKs have recently been identified in cucumber ${ }^{12}$; and 75 MAPKKKs, 12 MAPKKs and 16 MAPKs are present in the B. distachyon genome ${ }^{13}$. To date, few MAPK signaling cascades have been characterized in Arabidopsis. MEKK1 is active upstream of MKK1 and MPK4 in flagellin and ROS signaling ${ }^{14,15}$, and also plays a role upstream of MKK2 and MPK4, which can be activated during cold acclimation and contributes to the acquisition of freezing tolerance ${ }^{16}$. The MEKK1-MKK1/2-MPK4 cascade was shown to positively regulate defense responses against necrotrophic fungi while negatively regulating defenses against biotrophic pathogens ${ }^{17}$. MKK1/2-MPK4/6 cascades have been shown to play important roles in the response to salt and cold stress, as well as pathogen infection ${ }^{18,19}$. MKK3 displays an atypical MKK structure, which can act on MPK7 and MPK8 to mediate ROS signaling and regulate MPK6 in response to jasmonic acid ${ }^{20,21}$. In addition, the MEKK17/18-MKK3-MPK1/2/7/14 cascade is an ABA dependent MAPK pathway and plays a role in ABA stress signaling ${ }^{22}$. MKK4/5-MPK3/6 cascades play vital roles in plant

State Key Laboratory of Crop Genetics \& Germplasm Enhancement, Hybrid Cotton R\&D Engineering Research Center, Ministry of Education, Nanjing Agricultural University, Nanjing 210095, China. Correspondence and requests for materials should be addressed to W.G. (email: moelab@njau.edu.cn) 


\begin{tabular}{|l|l|l|l|c|c|c|c|c|c|}
\hline Name & \multicolumn{1}{|c|}{ ID $^{*}$} & Chr** $^{* *}$ & Published MKK & $\begin{array}{c}\text { Accession } \\
\text { number }\end{array}$ & $\begin{array}{c}\text { AA } \\
\text { length }\end{array}$ & pI & MW(Kd) & $\begin{array}{c}\text { Group } \\
\text { localization }\end{array}$ \\
\hline GrMKK1 & Gorai.009G033800.1 & D05(Chr09) & MKK1(HQ828075.1) & KX118695 & 361 & 5.49 & 40.09 & A & Cytoplasmic \\
\hline GrMKK2_1 & Gorai.001G013600.1 & D07(Chr01) & & & 362 & 6.19 & 40.23 & A & Out membrane \\
\hline GrMKK2_2 & Gorai.010G085300.1 & D06(Chr10) & & KX118696 & 364 & 8.71 & 40.91 & A & Out membrane \\
\hline GrMKK6 & Gorai.013G229600.1 & D13(Chr13) & & KX118697 & 354 & 6.28 & 39.93 & A & Outmembrane \\
\hline GrMKK3 & Gorai.007G075600.1 & D11(Chr07) & & KX118698 & 518 & 5.62 & 57.65 & B & Cytoplasmic \\
\hline GrMKK4 & Gorai.010G221800.1 & D06(Chr10) & MKK4(FJ966866) & KX118699 & 349 & 9.3 & 38.88 & C & Periplasmic \\
\hline GrMKK5 & Gorai.009G117800.1 & D05(Chr09) & MKK5(HQ637469.1) & KX118700 & 350 & 8.92 & 38.91 & C & Periplasmic \\
\hline GrMKK7 & Gorai.008G291700.1 & D12(Chr08) & & KX118701 & 323 & 8.4 & 36.35 & D & Cytoplasmic \\
\hline GrMKK9 & Gorai.008G291800.1 & D12(Chr08) & MKK9(HM989878.1) & & 321 & 7.63 & 36.23 & D & Cytoplasmic \\
\hline GrMKK10_1 & Gorai.003G184000.1 & D03(chr03) & & KX118702 & 322 & 7.1 & 35.74 & D & Cytoplasmic \\
\hline GrMKK10_2 & Gorai.008G225100.1 & D12(Chr08) & & & 320 & 7.09 & 35.42 & D & Cytoplasmic \\
\hline
\end{tabular}

Table 1. Genome-wide analysis of MKK genes in G. raimondii. *Sequence information from G. raimondii ${ }^{37}$. **Chromosome numbers D1 to D13 refer to chromosomes name of D subgenome in allotetraploid cultivated cotton species $^{38}$, and the names of 13 scaffolds from the G. raimondii genome ${ }^{37}$ are shown in brackets. ***Subcellular localization predicted using software (http://cello.life.nctu.edu.tw).

innate immunity ${ }^{23,24}$, and the MKK1-MPK3/6 and MKK4/5-MPK3/6 modules participate in stomatal development and stomatal dynamics ${ }^{25,26}$. MKK6-MPK4/11 cascades directly regulate cytokinesis and mitosis ${ }^{27}$, while MKK9-MPK3/6 cascades regulate ethylene signaling and camalexin biosynthesis, and may also play a role in leaf senescence ${ }^{28,29}$

Cotton is one of the most important economic crops in the world, and provides the world's leading natural textile fiber and considerable amounts of edible oil. Several MKKs have been reported in cotton. MKK1 is responsive to salt and drought stress: Ectopic expression of $M K K 1$ in $N$. benthamiana plants enhanced their salt and drought tolerance, but increased the transgenic plants' pathogen sensitivity ${ }^{30}$. MKK2 plays an important role in pathogen attack and in silencing MKK2-compromised cotton resistance to Verticillium wilt ${ }^{31}$. MKK4 plays an important role in abscisic acid-induced catalase 1 expression and $\mathrm{H}_{2} \mathrm{O}_{2}$ production ${ }^{32}$, while $M K K 5$ can be induced by pathogen infection, abiotic stresses and multiple defense-related signaling molecules. Overexpression of $M K K 5$ in $N$. benthamiana enhanced the plants' resistance to bacterial pathogens, but reduced their tolerance to salt and drought stresses ${ }^{33}$. A growing body of evidence suggests that there are fewer MKKs than MAPKKKs and MAPKs, suggesting that individual MKKs may function as bifurcation points and are likely to be involved in multiple MAPK cascades in response to a variety of stresses ${ }^{34}$. In addition to those described above, a total of 78 MAPKKK genes and 28 MAPK genes have been identified in the G. raimondii genome ${ }^{35,36}$. In spite of this, the systematical investigation of the MKK gene families in cotton and the verification of the interactions between MKK and MAPK members in MAPK cascades have not been studied. Here, we identified 11 MKK genes in the G. raimondii genome through database searches, and classified them according to their homology with those in Arabidopsis. We analyzed their chromosomal location, sequence phylogeny, genomic structure, and evolutionary mechanisms. We subsequently, investigated the expression level of MKK genes in different tissues and in response to different hormone, temperature and stress treatments in upland cotton. Furthermore, we cloned their corresponding orthologous MKK genes in G. hirsutum, examined the interaction profile of MKK-MAPK proteins using yeast two-hybrid ( $\mathrm{Y} 2 \mathrm{H})$ assays, and compared the interaction network and expression patterns of MKKs and MAPKs, which showed that several potential MAPK signaling modules were involved in the complicated cross-talk between hormones and abiotic stresses. Through this work, we can better understand signal-related stress and abiotic stress responses, as well as the corresponding molecular mechanisms regulated by MAPK cascades, in cotton. The data presented here lay a solid foundation for a deeper understanding of MAPK cascades and for future improvement of various defense responses using the signaling pathways in cotton breeding.

\section{Results}

Identification of MKK Genes in Cotton. To identify MKK genes in G. raimondii ${ }^{37}$, we used 18 MAPKK protein sequences, 10 from Arabidopsis and 8 from $O$. sativa, as direct queries to screen the G. raimondii genomic database (http://www.phytozome.net). As a result, a total of $11 \mathrm{MKK}$ genes were identified in G. raimondii. With the release of whole-genome sequence information of allotetraploid G. hirsutum $(\mathrm{Gh})^{38}$ and G. barbadense $(\mathrm{Gb})^{39}$, and diploid G. arboreum $(\mathrm{Ga})^{40}$, we further identified 20, 20 and 11 MKK genes from the three cotton species, respectively (Supplementary Dataset 1). Notably, the number of MKK genes in allotetraploid cotton is twice as many as in diploid cotton. Nine genes had one copy in the diploid G. raimondii and G. arboreum genomes, and two homologous genes (homoeologs from the A and D subgenomes) were found in the tetraploid G. hirsutum and G. barbadense genomes. The homoeologs of two were inconsistent, which might result from gene loss during their individual evolutionary processes or from assembly error in partial chromosomal regions, and needs to be further confirmed. These MKK genes were predicted to encode proteins from 320 to 518 amino acids in length, with putative molecular weights (MWs) ranging from 35.42 to $57.65 \mathrm{KDa}$ and isoelectric points (pIs) ranging from 5.49 to 8.92 (Table 1). To elucidate the chromosomal distribution of these MKK genes, we integrated 13 scaffolds of the G. raimondii genome ${ }^{37}$ (named as Chr. 1 to Chr. 13) with the reported high-density interspecific genetic map of allotetraploid cultivated cotton species, and confirmed that the 11 candidate MKK genes matched 


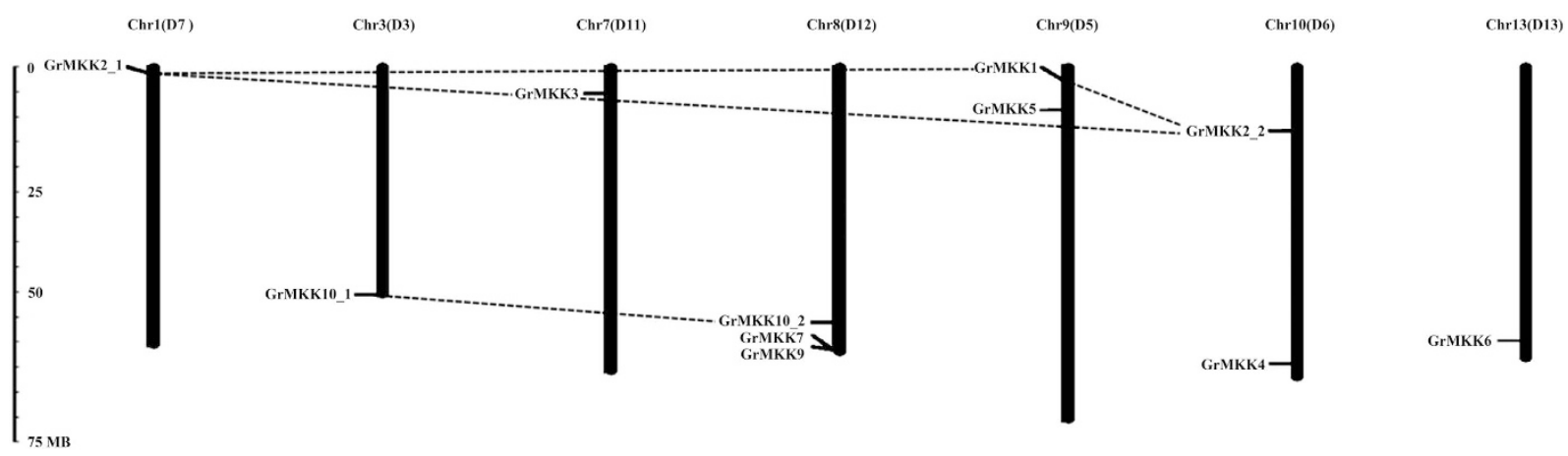

Figure 1. Chromosomal distribution of MKK genes in G. raimondii. Eleven candidate MKK genes were matched to 7 chromosome of the G. raimondii genome ${ }^{37}$. The nomenclature of MKK genes in G. raimondii were based on MKKs in A. thaliana. Lines were drawn to connect duplicated genes.
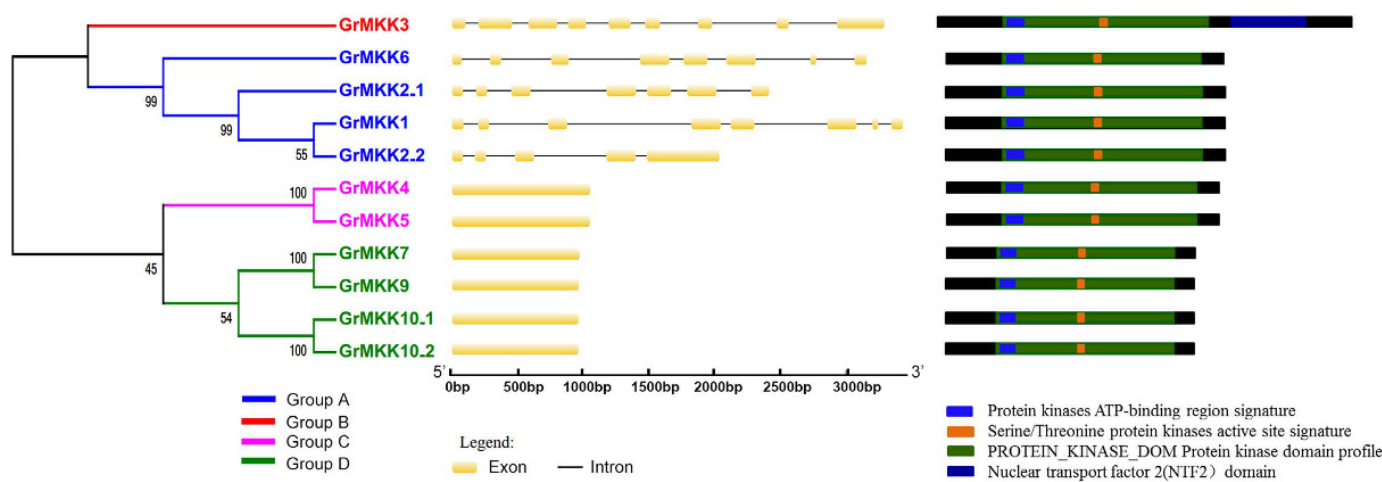

Figure 2. Phylogenetic analysis, intron-exon identification and structural comparison of 11 MKK genes in G. raimondii. A phylogenetic tree was constructed using MEGA 6 by ML method with 1000 bootstrap replicates. Intron and exon were represented by black line and orange box. Four conserved domains of each MKK gene were shown with blue, orange, green and dark blue, respectively.

to 7 chromosomes (Fig. 1). Each gene was named according its amino acid homology with that in Arabidopsis, and if two or more genes had the same homologs in Arabidopsis, they were distinguished by an extra number. Information on the MKK genes in G. raimondii, including their names, origins, chromosome locations, pIs, MWs and subcellular localizations, are shown in Table 1.

The G. raimondii genome underwent at least two rounds of genome-wide duplication ${ }^{37}$. To understand the mechanism of expansion of the $11 \mathrm{MKK}$ genes in G. raimondii, we investigated tandem and segmental duplication events of MKK gene family members on 7 chromosomes by genome synteny analysis. As shown in Fig. 1, five paralogs of $11 \mathrm{GrMKKs}$ were identified, including four segmental duplications (GrMKK1-GrMKK2_1, GrMKK1-GrMKK2_2, GrMKK2_1-GrMKK2_2 and GrMKK10_1-GrMKK10_2) and one tandem duplication (GrMKK7-GrMKK9). Furthermore, these paralogs were clustered together in the phylogenic tree and shared similar exon-intron structures (Fig. 2). These results indicate that segmental duplication events played significant roles in MKK gene expansion in the G. raimondii genome.

Multiple Alignment, Phylogenetic and Domain Analyses of GrMKKs. Alignment of amino acid sequences revealed that the GrMKKs contain 11 domains (I-XI; Supplementary Fig. 1). S/T-X5-S/T motifs in GrMKKs were located in the activation loop between the kinase subdomains VII and VIII. Phylogenetic analysis indicated that the GrMKKs could be divided into four major groups, with four members in group A, four in group $\mathrm{D}$, two in group $\mathrm{C}$, and only GrMKK3 in group $\mathrm{B}$. This is consistent with the phylogenetic relationship of MKKs in Arabidopsis (Fig. 2). Analysis of the exon positions and intron phases in MKK genes showed that the members in groups $\mathrm{C}$ and $\mathrm{D}$ had no introns and only GrMKK3 in group B contained nine exons. Compared with those in groups $\mathrm{B}, \mathrm{C}$ and $\mathrm{D}$, the MKKs in group $\mathrm{A}$ had a complex distribution of exons and introns, where GrMKK1 and GrMKK6 had eight exons, and GrMKK2_1 and GrMKK2_2 had seven and five exons, respectively. The diversity in the exon-intron structures among the different phylogenetic subgroups showed that duplication events were likely to have occurred historically, and the offspring genes evolved to have diverse exon-intron structures to accomplish different functions. Conserved domain analysis showed that all the GrMKKs contained three types of special subdomains; the Serine/Threonine protein kinases active site, an ATP binding site and a protein kinase domain. Moreover, GrMKK3 had a unique C-terminal NTF2-like domain, which may be essential for its nuclear localization (Fig. 2). 


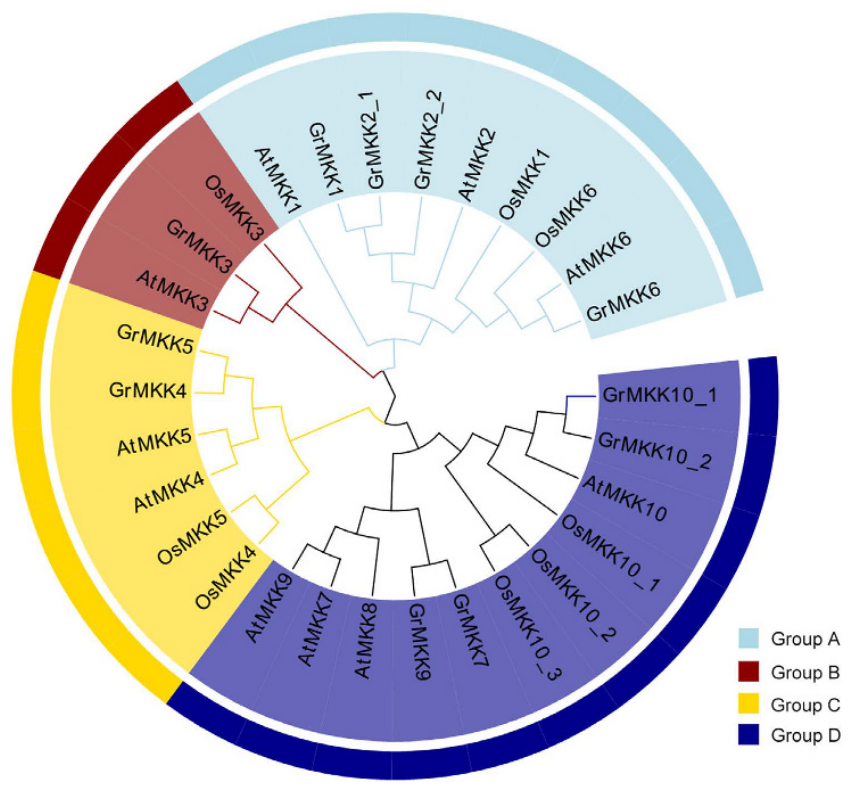

Figure 3. Phylogenetic tree of MKK family genes from G. raimondii, A. thaliana and O. sativa. Amino acid sequences were aligned using Clustal W in MEGA 6 software and ML method was used to perform phylogenetic analysis with 1,000 bootstrap replicates.

To further explore the evolutionary relationships between the MKKs in G. raimondii and those in other species, an un-rooted tree was constructed using 10 MKKs from Arabidopsis, 8 MKKs from O. sativa and 11 MKKs from G. raimondii. Phylogenetic analysis indicated that all of the MKKs from different species could be classified into the A, B, C and D groups (Fig. 3). No members of groups B and C, which contained MKK3, MKK4 and MKK5, were missing from the three species; In group A, there were nine MKKs, MKK1, MKK2 and MKK6 in Arabidopsis, MKK1, MKK6 in O. sativa, and MKK1, MKK2_1, MKK2_2 and MKK6 in G. raimondii; In Group D eleven MKKs, MKK7, MKK8, MKK9 and MKK10 in Arabidopsis, MKK10_1, MKK10_2, MKK10_3 in O. sativa and MKK7, MKK9, MKK10_1 and MKK10_2 in G. raimondii, were clustered together. Based on the phylogenetic analysis, MKK family members were conserved among dicots, however some in groups A and D were lost after the divergence of the monocots and dicots. As expected, most of the MKKs in G. raimondii were clustered more closely with those in Arabidopsis than O. sativa.

Spatial and Temporal Expression of MKK Genes in G. Hirsutum TM-1. Cultivated tetraploid cotton species differ greatly with respect to plant morphology as well as economic characteristics, including fiber production, oil content and stresses resistance. To explore the organ-specificity of MKK family members, we examined the transcripts abundance in different tissues of G. hirsutum TM-1, including roots, stems, leaves, petals, anthers, ovules and fibers, at three different developmental stages ( 0 days post-anthesis [dpa], $10 \mathrm{dpa}$ and $20 \mathrm{dpa}$ ). As shown in Supplementary Fig. 2, GhMKK1 and GhMKK2_2 in group A, GhMKK3 in group $\mathrm{B}$, and $G h M K K 5$ in group $\mathrm{C}$ were predominantly expressed in vegetative and reproductive organs, with the highest expression observed for GhMKK2_2 and GhMKK3 in all tissues and organs. GhMKK7 in group D was predominantly expressed in vegetative organs and had the highest expression levels in root and leaf tissues. GhMKK2_1 and GhMKK6 in group A were predominantly expressed in fibers at different development stages: GhMKK2_1 was most highly expressed in 20 dpa fibers, while GhMKK6 was preferentially expressed in 0 dpa ovules. GhMKK4 in group C was ubiquitously expressed in all tissues but was preferentially expressed in petals. GhMKK9, GhMKK10_1 and GhMKK10_2 in group D had very low or undetectable expression levels in all tested tissues. MKKs in the same group with high structure identity had diverse expression patterns in different tissues, indicating the functional diversity of duplicated genes. In contrast, however, the similar expression profile of genes from different groups suggested that these paralogs share similar functions.

Induced Expression Patterns of MKK Genes Under Multiple Phytohormone Treatments and Abiotic Stresses. MAPK cascades were not only involved in plant growth and development, but also played key roles in the control of plant responses to multiple environmental stimuli, including abiotic phytohormones and stresses. Here, we conducted qRT-PCR analyses to examine the expression levels of the MKK genes in response to three phytohormones (jasmonic acid [JA], abscisic acid [ABA] and salicylic acid [SA]). With the exception of $M K K 9$, the induced expression of all MKKs was detected (Fig. 4). After JA treatment, three MKK genes (GhMKK1, GhMKK2_2 and GhMKK6) in group A and two MKK genes (GhMKK7 and GhMKK10_1) in group D were up-regulated, reaching peaks at different time points. In addition, five MKK genes were significantly up-regulated after ABA treatment. Of them, the expression of GhMKK6 in group A and GhMKK10_1 in reached a peak at $1 \mathrm{~h}$, and GhMKK2_2 in group A, GhMKK5 in group C and GhMKK7 in group D, reached peaks at 6 or $8 \mathrm{~h}$ after treatment. Finally, GhMKK6 was induced and reached an expression peak at $24 \mathrm{~h}$, and all 


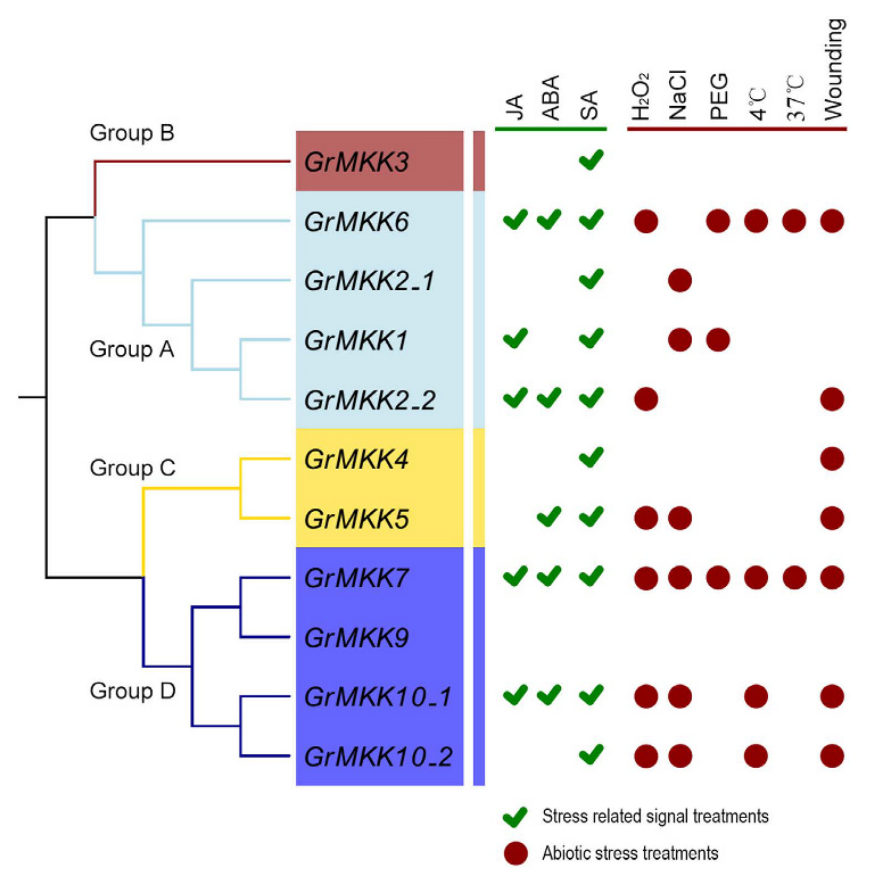

Figure 4. Expression pattern of MKK genes under signal and stress treatment. The tick (green color) indicated the MKKs induced under stress-related signal treatments, including JA, ABA and SA. The circle (dark red) indicated the MKKs induced under stresses treatment, including $\mathrm{H}_{2} \mathrm{O}_{2}, \mathrm{NaCl}, \mathrm{PEG}, 4^{\circ} \mathrm{C}, 37^{\circ} \mathrm{C}$ and wounding.

other MKK genes were significantly up-regulated and reached expression peaks $6 \mathrm{~h}$ after SA treatment. Detailed information on the expression patterns of each MKK under each treatment is shown in Supplementary Fig. 3.

The expression patterns of MKK genes following abiotic stress treatment were also analyzed in detail. We performed qRT-PCR to detect differences in their expression after six stress treatments (oxidative stress $\left[\mathrm{H}_{2} \mathrm{O}_{2}\right]$, salinity, drought, cold, heat and wounding) (Fig. 4). As a result, six MKK genes, two (GhMKK2_2 and GhMKK6) in group A, GhMKK5 in group C, and three (GhMKK7, GhMKK10_1 and GhMKK10_2) in group D, were significantly up-regulated after $\mathrm{H}_{2} \mathrm{O}_{2}$ treatment, and had diverse expression patterns. Also, six MKK genes, GhMKK1 and GhMKK2_1 in group A, GhMKK5 in group C, GhMKK7, and GhMKK10_1 and GhMKK10_2 in group D, were induced after $\mathrm{NaCl}$ treatment and had diverse expression patterns. Three MKK genes were induced by drought treatment; two (GhMKK1 and GhMKK6) in group A and GhMKK7 in group D. GhMKK7 was particularly highly induced and reached a peak at $6 \mathrm{~h}$. In addition, after low temperature treatment $\left(4^{\circ} \mathrm{C}\right), G h M K K 10 \_2$ was induced and reached a peak at $4 \mathrm{~h}$, and three other MKK genes (GhMKK6, GhMKK7 and GhMKK10_1) were induced and highly expressed after $12 \mathrm{~h}$. Moreover, only two MKK genes were induced and highly expressed upon exposure to high temperature conditions $\left(37^{\circ} \mathrm{C}\right)$ : GhMKK6 was induced and reached a peak at $8 \mathrm{~h}$, while GhMKK7 was up-regulated and highly expressed at 4 and $24 \mathrm{~h}$. Finally, seven of ten MKK genes were induced and reached peaks at $2 \mathrm{~h}$ or $6 \mathrm{~h}$ when the seedling leaves were cut with scissors; three in group $\mathrm{D}$ (GhMKK7, GhMKK10_1 and GhMKK10_2), two in group A (GhMKK6 and GhMKK2_2) and two in group C (GhMKK4 and $G h M K K 5)$. Further details of the expression patterns of individual genes under each treatment are shown in Supplementary Fig. 4.

Ten MKK genes were responsive to three hormone treatments. GhMKK2_2, GhMKK6, GhMKK7 and GhMKK10_1 were simultaneously induced and accumulated at high levels after all three hormone treatments. GhMKK1 and GhMKK5 were induced by two inducers, and GhMKK2_1, GhMKK3, GhMKK4 and GhMKK10_2 were only induced by SA treatment. All MKK genes, with the exception of GhMKK3, were induced by at least one of six stress treatments. GhMKK4 were only induced by wounding, GhMKK2_1 was induced by $\mathrm{NaCl}$, and GhMKK1 and GhMKK5 were induced by two or three abiotic stress treatments. GhMKK10_1, GhMKK10_2 and GhMKK6 were induced by four or five abiotic stress treatments. GhMKK7 was induced and expressed at high levels under six abiotic stress treatments (Table 2). These expression patterns suggest that MKKs respond to various hormones and carry out multiple physiological functions to help plants adapt to a variety of complex environmental challenges.

To investigate whether these duplicated paralog pairs were with the same expression patterns, we compared the expression profiles of the duplicated paralogs in different organs and under different stress treatments (Supplementary Table 1). There was no significant correlation between the expression of paralogs in different tissues and organs. However, the four paralogs showed the clear positive correlations in $\mathrm{ABA}, \mathrm{H}_{2} \mathrm{O}_{2}$ and SA stress-related signals, and GhMKK2_1 and GhMKK2_2 were positively correlated following JA treatment. Further, GhMKK1 was positively correlated with GhMKK2_2, and GhMKK2_1 was positively correlated with GhMKK2_2 under five abiotic stresses, GhMKK1 and GhMKK2_1 were positively correlated under three abiotic 


\begin{tabular}{|c|c|c|c|c|c|c|c|c|c|c|}
\hline \multirow[b]{2}{*}{ Gene } & \multirow[b]{2}{*}{ Group } & \multicolumn{3}{|c|}{ Signaling molecules } & \multicolumn{6}{|c|}{ Environmental stress factors } \\
\hline & & $\begin{array}{c}\text { JA } \\
(100 \mu \mathrm{M})\end{array}$ & $\begin{array}{c}\text { ABA } \\
(100 \mu M)\end{array}$ & $\begin{array}{c}\text { SA } \\
(10 \mathrm{mM})\end{array}$ & $\begin{array}{c}\mathrm{H}_{2} \mathrm{O}_{2} \\
(10 \mathrm{mM})\end{array}$ & $\begin{array}{c}\text { Salt } \\
(200 \mathrm{mM})\end{array}$ & $\begin{array}{c}\text { PEG6000 } \\
(20 \%)\end{array}$ & $4^{\circ} \mathrm{C}$ & $37^{\circ} \mathrm{C}$ & Wounding \\
\hline MKK1 & A & $* *$ & $*$ & $* *$ & $*$ & $* *$ & $* *$ & - & - & $*$ \\
\hline MKK2_1 & A & $*$ & $*$ & $* *$ & $*$ & $* *$ & $*$ & - & - & $*$ \\
\hline MKK2_2 & A & $* *$ & $* *$ & $* *$ & $* *$ & - & $*$ & $\mathrm{D}$ & $\mathrm{D}$ & $* *$ \\
\hline MKK6 & A & $* *$ & $* *$ & $* *$ & $* *$ & $*$ & $* *$ & $* *$ & $* *$ & $* *$ \\
\hline MKK3 & B & $*$ & $\mathrm{D}$ & $* *$ & $*$ & $\mathrm{D}$ & - & $\mathrm{D}$ & $\mathrm{D}$ & $\mathrm{D}$ \\
\hline MKK4 & $\mathrm{C}$ & - & $*$ & $* *$ & $\mathrm{D}$ & $*$ & - & - & - & $* *$ \\
\hline MKK5 & $\mathrm{C}$ & - & $* *$ & $* *$ & $* *$ & $* *$ & $*$ & $*$ & $*$ & $* *$ \\
\hline MKK7 & $\mathrm{D}$ & $* *$ & $* *$ & $* *$ & $* *$ & $* *$ & $* *$ & $* *$ & $* *$ & $* *$ \\
\hline MKK10_1 & $\mathrm{D}$ & $* *$ & $* *$ & $* *$ & $* *$ & $* *$ & $*$ & $* *$ & $\mathrm{D}$ & $* *$ \\
\hline MKK10_2 & $\mathrm{D}$ & $*$ & - & $* *$ & $* *$ & $* *$ & $\mathrm{D}$ & $* *$ & $\mathrm{D}$ & $* *$ \\
\hline
\end{tabular}

Table 2. Expression profiles of MKK genes under different stress treatments in cotton. Note: For hormone treatments, the leaves of seedlings were harvested at $0,0.5,1,2,4,6,8,10,12$ and $24 \mathrm{~h}$ after treatment; For the environmental stress factor treatments, the leaves of seedlings were harvested at $0,0.5,1,2,4,6,8,10,12$ and $24 \mathrm{~h}$ after treatment; “**” and "*” indicate significant difference at $\mathrm{P}<0.01$ and $\mathrm{P}<0.05$, respectively; "-_" represents no change and weak upregulation; " $D$ ” represents significant reduction in MKK gene expression after treatment; The Student's t-test was performed between treated samples and untreated samples.

stresses $\left(4^{\circ} \mathrm{C}, 37^{\circ} \mathrm{C}\right.$, wounding), and GhMKK10_1 and GhMKK10_2 were positively correlated under $\mathrm{NaCl}$ and $37^{\circ} \mathrm{C}$ treatments. These results indicate that the duplicated MKK gene pairs had similar functional responses to different stresses, although their expression differed in different tissues and organs.

Interactions Between MKK and MAPK Protein Family Members. Since the nature of protein kinase activity depends on their direct physical encounters, we conducted a comprehensive directed yeast two-hybrid $(\mathrm{Y} 2 \mathrm{H})$ assay to define the interactions between the last two kinases in the MAPK cascade in cotton. Besides the 28 MAPK genes reported previously ${ }^{36}$, we further confirmed 56, 52 and 28 MAPK genes from G. hirsutum, G. barbadense and G. arboreum, respectively (Supplementary Dataset 2). Due to the high similarity (more than 97\%) in the amino acid sequences (Supplementary Table 2) of MKK and MAPK homoeologs in the A- and D-subgenomes, we performed PCR-based cloning for anyone of the MKK homoeologs. In total, eight MKK genes were cloned and sequenced to confirm their complete open reading frame (GenBank accession numbers: KX118695-KX118702) (Table 1). Combined with the 21 MAPK genes cloned previously ${ }^{36}$, we constructed eight MKKs and 21 MAPKs into DNA binding domain and activation domain plasmid vectors, respectively. Each MKK protein was found to have a distinct auto-activation activity in yeast. To solve this issue, Aureobasidin A (ABA) was added to selective medium and the auto-activation of MKKs was inhibited completely (Supplementary Fig. 5).

Each MKK-MAPK pair was individually co-transformed into yeast cells. Colonies were tested on quadruple dropout medium (SD/-Ade/-His/-Leu/-Trp) and subsequently on quadruple dropout medium supplemented with X- $\alpha-$ Gal and ABA. A total of 63 interactions were identified in the $\mathrm{Y} 2 \mathrm{H}$ assay (Fig. 5). In the MKK-MAPK interaction pairs, eight of the MKK proteins were detected to interact with at least three MAPK proteins. GhMKK1, GhMKK2_2 and GhMKK6 in group A interacted with GhMPK3, GhMPK6 and GhMPK18. GhMKK1 and GhMKK2_2 also interacted with GhMPK12, GhMPK19, GhMPK23 and GhMPK27; and GhMKK1 and GhMKK6 interacted with GhMPK16. We also found that the genes that were duplicates of GhMKK1/2_2 had different interactions. GhMKK1 interacted with GhMPK5, GhMPK7, GhMPK15, GhMPK16, GhMPK20 and GhMPK22, while its sister, GhMKK2_2, did not show any affinity to these proteins, instead, it was detected to interact with GhMPK14, indicating the functional divergence of paralogous genes during the evolutionary process. GhMKK3 interacted with the MAPKs in group C (GhMPK5, GhMPK8, GhMPK14, GhMPK20, GhMPK23, GhMPK25), and also interacted with GhMPK16, GhMPK19 and GhMPK22. Both GhMKK4 and GhMKK5 belong to group C, however, they interacted with different proteins. GhMKK4 interacted with GhMPK8, GhMPK16 and GhMPK20; while GhMKK5 interacted with GhMPK3, GhMPK6, GhMPK12, GhMPK13, and GhMPK27. GhMKK7 interacted with GhMPK8, GhMPK14, GhMPK16 and GhMPK25 (Fig. 5). Additionally, although expressed at a low level in all tissues analyzed, GhMKK10_1 was detected to interact with 17 of the 21 MAPK genes in vitro. It did not interact with GhMPK5, GhMPK10, GhMPK14 and GhMPK25 (data not shown), therefore its functional role needs to be further clarified.

Of the 63 MKK-MAPK interactions detected, 23 have been reported in Arabidopsis, indicating that these interactions are reproducible in different species. The remaining 40 interaction pairs have not been reported in Arabidopsis and are novel interaction combinations found in cotton. We also found that each MKK protein was able to interact with at least three MAPK proteins, and multiple MKKs individually interacted with one MAPK, for example, GhMPK3 and GhMPK6 were able to interact with GhMKK1, GhMKK2_2, GhMKK5 and GhMKK6, indicating function universality in the MAPK signal pathway (Fig. 6).

Comparison of the Expression Patterns Between MKK and MAPK Genes. The last components of the MAPK cascade (MAPK) in cotton development and responses to abiotic and biotic stress were characterized in our laborator ${ }^{36}$. In the study, the majority of MKK genes in cotton were generally responsive to stress-related signals and abiotic stress treatments. Integrating these data, we compared the expression of MKKs 

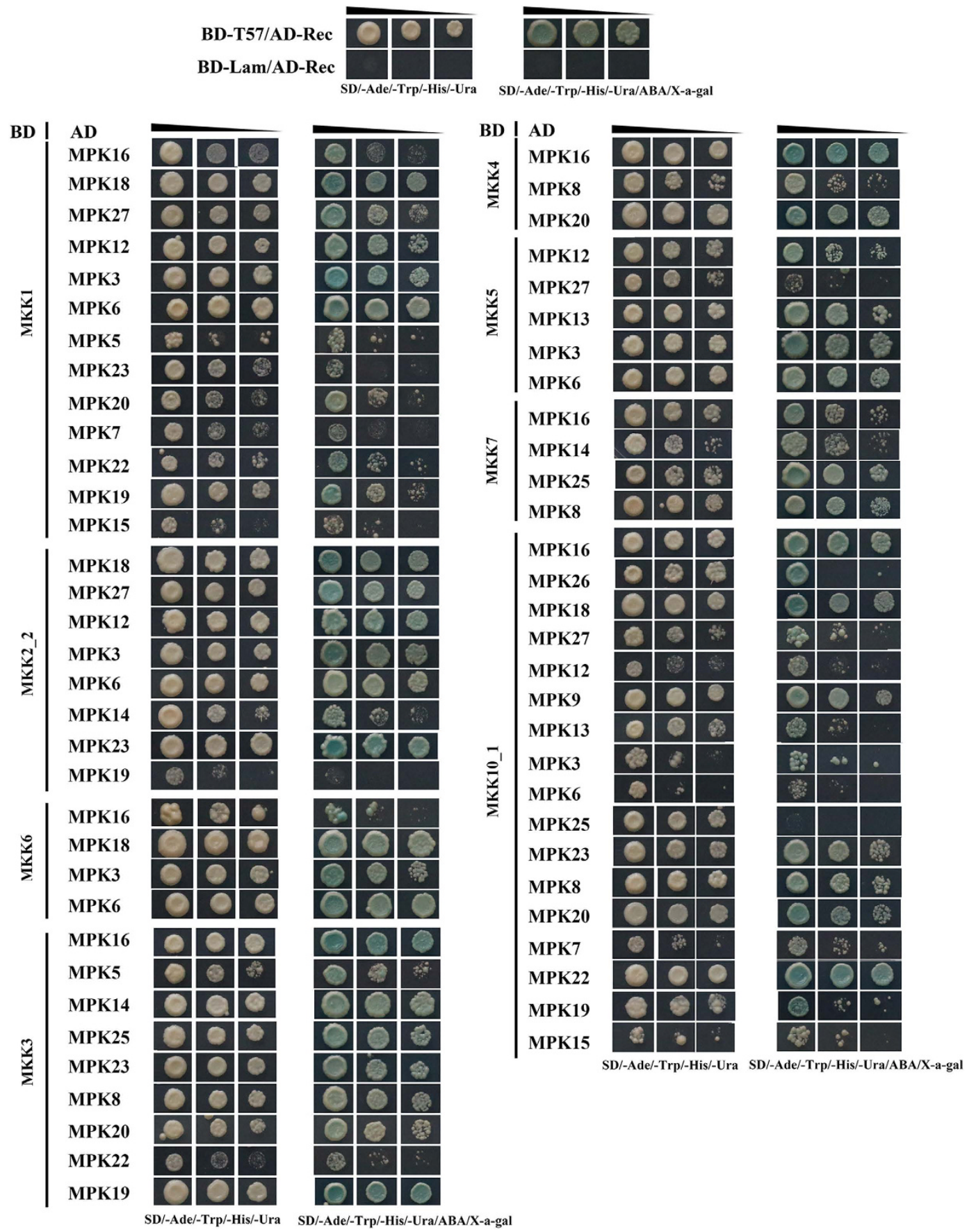

Figure 5. Comparative yeast two-hybrid interaction analyses of MKK with MAPKs. Interactions between selected MKKs and MAPKs. Yeasts harboring the indicated plasmid combinations were grown on selective medium SD/-Ade/-Trp/-His/-Ura, positive interactions were examined by addition of Aureobasidin A and X- $\alpha$ -gal. Positive (pGBKT7-53 + pGADT7-rec) and negative (pGBKT7-Lam+pGADT7-rec) controls. Repeated experiments showed similar results.

and MAPKs in various tissues following stress-related signals and abiotic stress. In general, the expression levels of MKKs were lower than those of MAPKs in 22 G. hirsutum TM-1 tissues (Supplementary Fig. 6, Supplementary Dataset 3). Comparison of the expression of MKK and MAPK genes under stress-related signal and abiotic treatments revealed that MAPK genes were more sensitive to stress than MKK genes (Supplementary Figs 7-8, Supplementary Datasets 4-5). However, both MKK and MAPK genes were more sensitive to stress-related signals than abiotic stress.

To further characterize the biological functions of the confirmed MKK-MAPK interaction pairs, we analyzed their responses to signal and stress treatments. Integration analysis of the interaction networks and expression patterns of MKK-MAPK family members revealed 13 potential MAPK signaling modules that are involved in the complicated cross-talk between hormones and abiotic stresses (Fig. 7). Genes 


\section{Group A \\ Group B \\ Group C}

(AtMPK3) (AtMPK6) (AtMPK6) (AtMPK4) (AtMPK11) (AtMPK5) (AtMPKI3) (AtMPKI) (AtMPK2) (AtMPK7) (AtMPK7) (AtMPK7) (AtMPK3)

MPK13 MPK3 MPK6 MPK16 MPK18 MPK27 MPK12 MPK5 MPK14 MPK8 MPK20 MPK23 MPK25
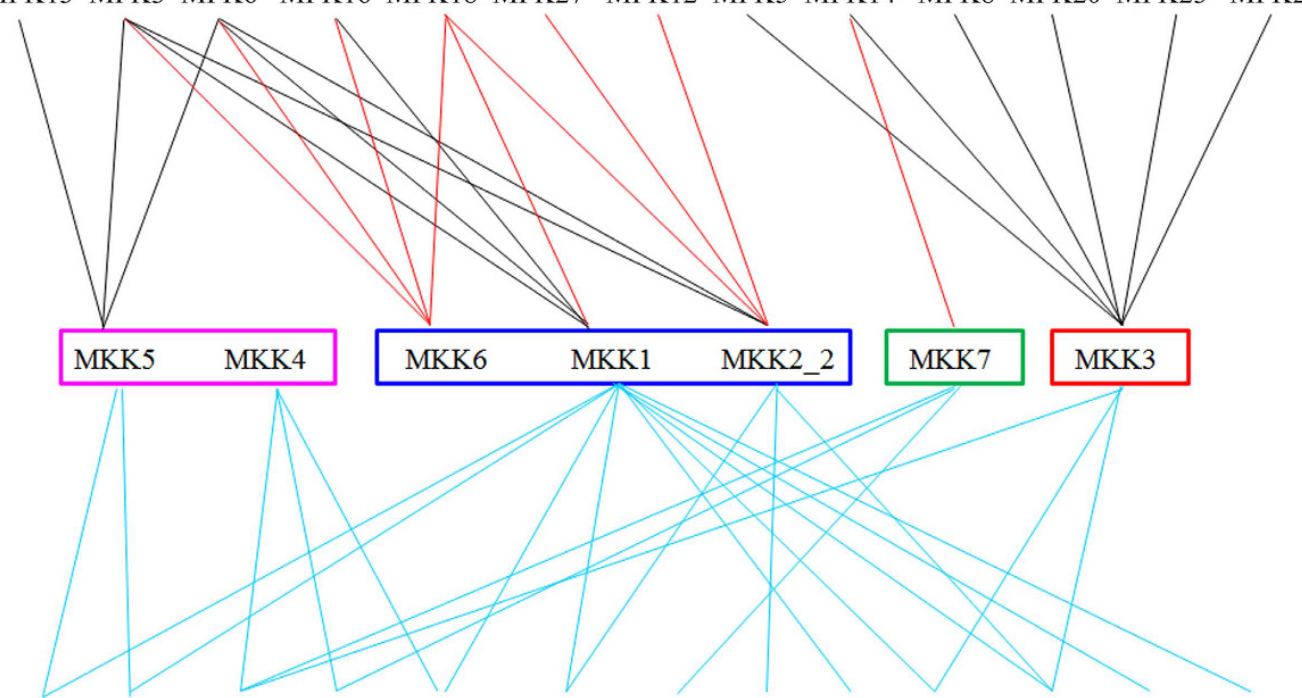

MPK12 MPK27 MPK16 MPK8 MPK20 MPK23 MPK25 MPK14 MPK5 MPK22 MPK19 MPK7 MPK15

(AtMPK13) (AtMPK5) (AtMPK4) (AtMPK7) (AtMPK7) (AtMPK7) (AtMPK7) (AtMPK2) (AtMPKI) (AtMPK9) (AtMPK20) (AtMPK17) (AtMPK19)
Group B
Group C
Group D

Figure 6. MKK-MAPK interaction in cotton compared to those in Arabidopsis. All the lines indicated the confirmed interactions of MKK-MAPK in cotton. Black lines indicated the interactions reported with confirmed function under stress in Arabidopsis, which was also identified in cotton. Red lines indicated the shared interactions in Arabidopsis and cotton, but no functions were reported in Arabidopsis. Blue lines indicated the interactions only confirmed in cotton without reported in Arabidopsis.

involved in GhMKK1-GhMPK15/16/23 interactions were all induced by JA, SA, NaCl and PEG. In the GhMKK2_2-GhMPK6/12 modules, the transcript levels of GhMKK2_2, GhMPK6 and GhMPK12 were increased following JA, ABA, SA, $\mathrm{H}_{2} \mathrm{O}_{2}$ and wounding treatments. All of the GhMKK3-GhMPK5/22/23/25 interactions were induced by SA. Both genes involved in the GhMKK4-GhMPK16 interaction were induced by SA and wounding, and both genes involved in the GhMKK5-GhMPK6 interaction were induced by ABA, SA, $\mathrm{H}_{2} \mathrm{O}_{2}, \mathrm{NaCl}$ and wounding. In addition, in the GhMKK7-GhMPK25 module, GhMKK7 and GhMPK25 were induced by all tested signal-related and abiotic stresses. Taken together, these data highlight the important role of post-transcriptional regulation of MKK-MAPK signaling cascades and the complicated cross-talk between hormones and abiotic stresses.

\section{Discussion}

Characterization of MKKs in G. Raimondii and Their Evolution. Based on the genome scans of several plant genomes, MKK family genes have been systematically investigated in Arabidopsis ${ }^{5}$, O. sativa ${ }^{41}$, tomato ${ }^{8}$, maize $^{10}$, cucumber ${ }^{12}$ and B. distachyon ${ }^{13}$. In the current study, a total of $11 \mathrm{MKKs}$ from G. raimondii were identified and classified into four groups (A-D) according to their phylogenetic clades, which were similar to those reported previously ${ }^{5}$. The MKKs in groups B and C, MKK3, MKK4 and MKK5, were conserved in three species, Arabidopsis, O. sativa and G. raimondii. Group A and D MKK genes in G. raimondii were expanded in comparison with that in Arabidopsis, whereas MKK8 in group D in Arabidopsis was not detected in G. raimondii. This indicates that most MKK family members were conserved in dicots, while some members belonging to groups $\mathrm{A}$ and $\mathrm{D}$ were lost after the divergence of the monocots and dicots (Fig. 3). In addition, synteny analysis of the G. raimondii genome indicated that the MKK family mainly resulted from segmental duplication, which is consistent with the evolution of the MAPK family ${ }^{36}$.

Functional Divergence of MKKs During Plant Growth and Development. Increasing evidence shows that MKK genes are involved in plant growth and development, and play key roles in the control of plant responses to abiotic stresses, biotic stresses and phytohormones ${ }^{42,43}$. For example, AtMKK4 and AtMKK5 control various aspects of stomatal development ${ }^{44}$, AtMKK6 plays an important role in meiotic cytokinesis during pollen development ${ }^{45}$, AtMKK7 has been shown to negatively regulate polar auxin transport ${ }^{46}, A t M K K 9$ plays an important role in leaf senescence ${ }^{28}$ and $O s M K K 4$ is a factor of seed/grain size and influences grain size in rice ${ }^{47}$. In the present study, we observed different expression patterns of MKKs in vegetative organs and reproductive organs in cotton. Two genes were constitutively expressed at high levels in both vegetative and reproductive organs. Two and three genes were expressed at high levels in vegetative organs and reproductive organs, respectively, and the 


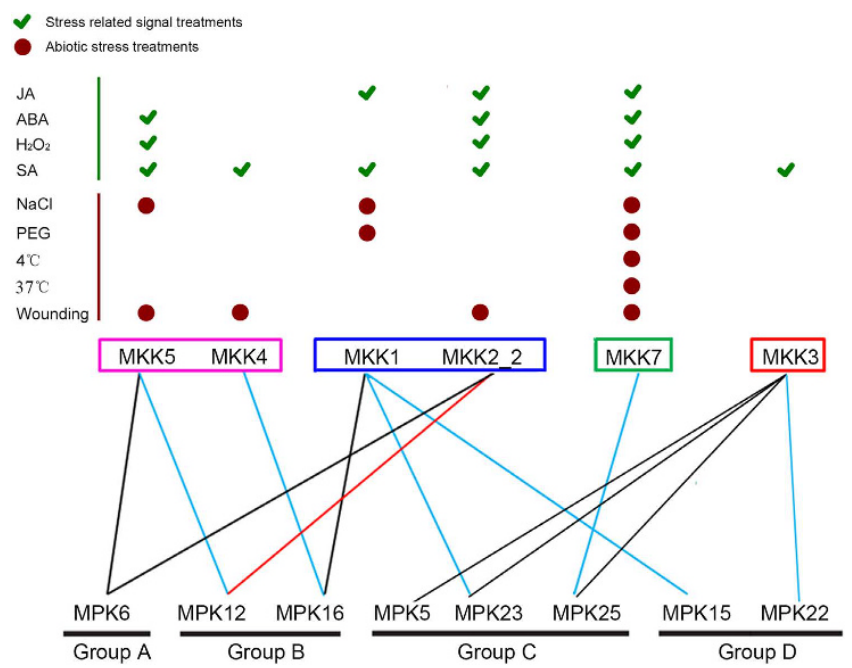

Figure 7. Integrated module of MKK-MAPK interactions in response to various treatments. The tick (green color) indicated the MKK-MAPK interactions commonly induced in stress-related signal treatments, including JA, ABA and SA. The circle (dark red) indicated the MPK-MPKK interactions commonly induced in stress treatment, including $\mathrm{H}_{2} \mathrm{O}_{2}, \mathrm{NaCl}, \mathrm{PEG}, 4^{\circ} \mathrm{C}, 37^{\circ} \mathrm{C}$ and wounding. Black lines indicated the interactions reported with confirmed function under stress in Arabidopsis, which was also identified in cotton. Red lines indicated the shared interactions in Arabidopsis and cotton, but no functions were reported in Arabidopsis. Blue lines indicated the interactions newly confirmed in cotton without reported in Arabidopsis.

remaining genes were expressed at low levels in all tested organs. The tissue- or organ-specific MKK expression patterns indicate their functional divergence during plant development and growth.

Accumulating evidence demonstrates that members of the MKK gene family in Arabidopsis, rice and other species function in response to abiotic and biotic stresses ${ }^{3}$. In Arabidopsis, MKK1/2 plays important roles in the response to salt and cold stress and to pathogen attack ${ }^{16,19}$. MKK4/5 plays a vital role in plant innate immunity ${ }^{23,24}$. $M K K 3$ is an essential component of ROS metabolism and is involved in JA and ABA stress signaling ${ }^{20,22,48,49}$. $M K K 7$ is involved in generating the mobile signal for SAR ${ }^{50}$. In cotton, $M K K 1$ contributes to the tolerance of salt and drought stress, and therefore is a crucial regulator of the response to environmental stresses ${ }^{30}$. MKK4 participates in regulating abscisic acid, gibberellin and hydrogen peroxide signaling ${ }^{32}$. MKK5 plays a negative role in the response to salt and drought stresses ${ }^{33}$. In our study, all 10 detected MKKs showed differential expression patterns in response to stress-related signals and abiotic stresses (Fig. 4). All MKK genes were induced by at least one stress-related signal treatment. $40 \%$ of the MKK genes were induced by three stress-related signals, and $20 \%$ was induced by two stress-related signals. The remaining genes were responsive to SA. Furthermore, GhMKK7 was responsive to six abiotic stresses, GhMKK6 was responsive to five abiotic stresses, GhMKK5 was responsive to three abiotic stresses, and $20 \%$ of MKK genes were responsive to four and two abiotic stresses, respectively. $20 \%$ were responsive to one of the abiotic stresses tested. In addition, GhMKK3 was insensitive or down-regulated in response to abiotic stresses. Taken together, these results show that MKKs are generally responsive to stress-related signals and abiotic stress treatments, and play an important role in the responses to environmental stresses. This highlights the extent of cross-talk that exists between stress-related signals and abiotic stress.

Integration analysis of the interaction levels and expression patterns of MKKs and MAPKs. Plant MAPKs regulate numerous physiological responses by interacting with upstream and downstream protein components. To date, the most extensively characterized MKK-MAPK interaction partners are from Arabidopsis ${ }^{51}$, O. sativa ${ }^{6,52}$, maize ${ }^{10}$, canola ${ }^{53}$, watermelon ${ }^{54}$ and $B$. distachyon ${ }^{13}$, and these have elucidated the cross-talk between different signaling pathways. Reports on MKK-MAPK interactions in cotton are limited. Here, we systematically analyzed interactions between eight MKKs and 21 MAPKs using the Y2H system. In addition to those of GhMKK10_1, a total of 46 interactions were identified, as shown in Fig. 6. We found 23 MKK-MAPK interactions that were consistent with those reported previously in Arabidopsis, and the function of 14 of these MKK-MAPK interaction pairs has been elucidated. In Arabidopsis, MKK1/2-MPK4/6 cascades have previously been shown to play important roles in the response to salt and cold stress and to pathogen attack ${ }^{18,19}$, which is consistent with our observation that GhMKK1/2_2 significantly interacted with GhMPK3/6, and that GhMKK1 interacted with GhMPK16, and the orthologous of relationship are as shown in Fig. 6. AtMKK3 has been reported to act on AtMPK7 and AtMPK8 to mediate ROS signaling and to regulate AtMPK6 in response to JA ${ }^{20,21}$. In addition, AtMKK3 plays a role in ABA stress signaling, which subsequently stimulates the activity of the group C MAPKs, including AtMPK1, AtMPK2, AtMPK7 and AtMPK14 ${ }^{22}$. We also found that GhMKK3 significantly interacted with group C MAPKs, which including GhMPK5, GhMPK8, GhMPK14, GhMPK20, GhMPK23 and GhMPK25. AtMEKK1-AtMKK4/5-AtMPK3/6 cascades play vital roles in the plant innate immunity ${ }^{23,24}$. We also revealed that GhMKK5 significantly interacted with GhMPK13, GhMPK3 and GhMPK6. The interacting 
pairs and their associated complicated linear MAPK pathways function in regulating plant immune responses and other processes in cotton and are worth analyzing further. There were nine other interaction patterns (red lines in Fig. 6), consistent with Arabidopsis homologs: GhMKK1 interacted with GhMPK18; GhMKK2_2 interacted with GhMPK12, GhMPK18 and GhMPK27; GhMKK6 interacted with GhMPK3, GhMPK6, GhMPK16 and GhMPK18; and GhMKK7 interacted with GhMPK14. However, no function was reported for any of these interactions, therefore their functional roles are also worth clarifying further. In addition, 23 interactions differed from the previous observations in Arabidopsis. For example, GhMKK1 interacted with GhMPK5, GhMPK7, GhMPK12, GhMPK15, GhMPK19, GhMPK20, GhMPK22, GhMPK23 and GhMPK27; GhMKK2_2 interacted with GhMPK14, GhMPK19 and GhMPK23; GhMKK3 interacted with GhMPK16, GhMPK19 and GhMPK22; GhMKK4 interacted with GhMPK8, GhMPK16 and GhMPK20; GhMKK5 interacted with GhMPK12 and GhMPK27; and GhMKK7 interacted with GhMPK8, GhMPK16 and GhMPK25 (Fig. 6). These new interaction pairs in cotton imply an inherent difference in signaling pathways between Arabidopsis and cotton, and highlight the necessity to explore the MAPK-mediated signaling pathways for use in genetic investigations and breeding in cotton.

MAPK modules are known to be involved in various stress-related signals and abiotic stress responses. In Arabidopsis, MKK1/2 has previously been shown to play important roles in the response to salt and cold stress ${ }^{18,19}$. In our study, the GhMKK1-GhMPK15/16/23 interactions were induced by JA, SA, NaCl and PEG. GhMKK1 was found to play an important role in the tolerance of salt, drought and pathogen attack, and GhMPK7 (a paralog of GhMPK23) has a role in broad spectrum disease resistance ${ }^{55}$, suggesting that the GhMKK1-GhMPK15/16/23 interactions might be involved in salt and drought stress as well as defense against pathogens. All of the GhMKK2_2-GhMPK6/12 modules were induced JA, ABA, SA, $\mathrm{H}_{2} \mathrm{O}_{2}$ and wounding, and silencing of GhMKK2 compromises the resistance to cotton pathogens ${ }^{31}$, implying that GhMKK2_2-GhMPK6/12 plays an important role in the response to pathogen attack. AtMKK3 is an essential component of ROS metabolism and is involved in JA and ABA stress signaling ${ }^{20-22}$. In our study, GhMKK3-GhMPK5/22/23 interactions were induced by SA, suggesting that this MAPK cascade may be involved in generating the mobile signal for SAR in cotton. MKK4/5-MPK3/6 cascades play vital roles in innate immunity in Arabidopsis ${ }^{23,24}$. However, this is not the case for $M K K 4$ and $M K K 5$ in cotton; GhMKK4 plays an important role in abscisic acid, gibberellin and hydrogen peroxide signaling ${ }^{32}$. GhMKK5 can be induced by pathogen infection, abiotic stresses and multiple defense-related signal molecules ${ }^{33}$, implying that MKK4 and MKK5 may have different interaction substrates, and their functions are slightly different ${ }^{32,33}$. In our study, GhMKK4 and GhMKK5 both belonged to group C, but they interacted with different MAPKs. In the GhMKK4-GhMPK16 interaction, both genes were induced by SA and wounding, indicating that they are likely involved in the response to wounding stress and in SA signaling pathways. In the GhMKK5-GhMPK6 interaction, both genes were induced by ABA, SA, $\mathrm{H}_{2} \mathrm{O}_{2}, \mathrm{NaCl}$ and wounding, which suggests that the interaction might be involved in multiple signaling pathways and the response to salt stress. AtMKK7 has been shown to negatively regulate polar auxin transport and is involved in generating the mobile signal for SAR ${ }^{46,50}$. In the GhMKK7-GhMPK25 module, GhMKK7 and GhMPK25 were induced by all signal-related stresses and abiotic stresses. GhMPK7 (a paralog of GhMPK25) has a role in broad spectrum disease resistance ${ }^{55}$, and we also demonstrated that GhMPK25 is an important component of cotton resistance to V. dahliae ${ }^{36}$, which suggests that GhMKK7-GhMPK25 might be involved in multiple signaling pathways and responses to stress.

In summary, we performed the first comprehensive investigation of the MKK gene family in cotton involving in the analysis of sequence phylogeny, genomic structure, chromosomal location and adaptive evolution. The temporal and spatial expression patterns in vegetative and reproductive organs in response to stress-related signals and abiotic stresses revealed that MKKs may play a crucial role in driving evolutionary novelty and adaptation to new environments. Genome-wide MKK-MAPK interaction pairs were systematically identified in cotton, and 40 new interaction pairs were identified. Combining MKK-MAPK interaction pairs with their expression patterns further revealed 13 potential MAPK signaling modules involved in the complicated cross-talk between hormones and abiotic stresses. Our study is the first to systematically elucidate the relationship of the last two kinases of the MAPK cascade in different organs and under different stress conditions and this provides an important foundation for the further functional dissection of the last two kinases of the MAPK cascade for utilization in cotton breeding.

\section{Methods}

Characterization of MKK Genes in G. Raimondii. The MKKs in Arabidopsis and O. sativa were used as queries to search for putative MKK proteins against the G. raimondii genome ${ }^{37}$ database at http://www.phytozome.net. The PFAM (http://pfam.sanger.ac.uk) and SMART (http://smart.emblheidelberg.de/) databases were employed to detect conserved domains. The isoelectric points and molecular weights of the MKK proteins were predicted on the ExPASy proteomics server (http://expasy.org/). The subcellular localization of each MKK was analyzed using the CELLO v2.5 server (http://cello.life.nctu.edu.tw/). Sequence alignment was carried out by the Clustal X program and a phylogenetic tree was constructed by the Maximum likelihood (ML) method in MEGA6. Mapping of MKK genes was performed using MapInspect (http://www.plantbreeding.wur.nl/UK/software_mapinspect.html). Segment duplicates were identified using Plant Genome Duplication Database (http:// chibba,agtec.uga.edu/duplication), tandem duplicates were defined as genes separated by five or fewer genes. The exon/intron structures of individual MKK genes were determined by aligning the cDNA sequences to their corresponding genomic DNA sequences.

Transcriptome Data of the MKK and MAPK Family Genes in G. Hirsutum. The RNA-Seq data from distinct tissues have been previously reported in TM-1 genome sequencing research ${ }^{38}$, which was downloaded from http://mascotton.njau.edu.cn and NCBI database SRA: PRJNA248163. All these tissues include the root, stem and leaves of two week-old plants; torus, petal, stamen, pistil and calycle dissected from whole mature 
flowers; fiber bearing ovule at $-3,-1,0,1,3$ DPA, ovules at 5, 10, 20, 25 and 35 DPA, and fibers at 5, 10, 20 and 25 DPA. The fragments per kilobase of exon per million fragments (FPKM) was used to estimate the gene expression level of MKK and MAPK family genes in distinct tissue of G. hirsutum.

Plant materials and Treatments. G. hirsutum L. cv. Jinmain 19, which exhibits a high tolerance to abiotic stress, was used for the abiotic stress treatments. Cotton seedlings (G. hirsutum L.cv. Jinmian19) were grown in a growth chamber under greenhouse conditions at $28^{\circ} \mathrm{C}$ under a $16 \mathrm{~h} \mathrm{light} / 8 \mathrm{~h}$ dark cycle. Three-week-old cotton seedlings were used for the following treatments. For signaling substance treatments, leaves were sprayed with $100 \mu \mathrm{M} \mathrm{JA}, 100 \mu \mathrm{M}$ ABA or $10 \mathrm{mM} \mathrm{SA}$ ( $\mathrm{ddH}_{2} \mathrm{O}$ as a solvent control). For oxidative stress, leaves were sprayed with $10 \mathrm{mM} \mathrm{H}_{2} \mathrm{O}_{2}\left(\mathrm{ddH}_{2} \mathrm{O}\right.$ as a solvent control). For the salt and drought treatments, the roots of cotton seedlings were irrigated with $200 \mathrm{mM} \mathrm{NaCl}$ and $20 \% \mathrm{PEG}$, respectively ( $\mathrm{ddH}_{2} \mathrm{O}$ as a mock control). For temperature stress treatments, the seedlings were placed in a growth chamber at a high temperature $\left(37^{\circ} \mathrm{C}\right)$ or a low temperature $\left(4^{\circ} \mathrm{C}\right)$ $\left(28^{\circ} \mathrm{C}\right.$ as a mock control). Seedling leaves were cut with scissors for wound treatment. The leaves were harvested at the appropriate time points (triplicate samples were collected at each time point $[n=3$ seedlings]). All tissues were flash-frozen in liquid nitrogen and stored at $-70^{\circ} \mathrm{C}$ for further analysis.

Extraction of Total RNA. Cotton total RNA was extracted from leaves and roots using a Biospin plant Total RNA extraction kit (BioFlux) according to the manufacturer's protocol. RNA was then treated with Dnase I (Invitrogen, http://www.invitrogen.com) to remove genomic DNA. Purified RNA ( $2 \mu \mathrm{g})$ was subsequently reverse transcribed using a first-strand cDNA synthesis kit according to the manufacturer's instructions. The primer pairs used for real-time PCR were designed using Beacon Designer 7.0 according to cotton MKK gene sequences and synthesized commercially (Supplementary Dataset 6). The His3 (AF024716) gene was used as a control. Real-time PCR was performed on an ABI7500 Real time PCR system (Applied Biosystems, USA) using SYBR Green (Roche, Switzerland) with three replicates. The PCR program was as follows: initial denaturation at $95^{\circ} \mathrm{C}$ for $10 \mathrm{~min}, 40$ cycles of denaturation at $95^{\circ} \mathrm{C}$ for $15 \mathrm{~s}, 60^{\circ} \mathrm{C}$ for $15 \mathrm{~s}$ and $72^{\circ} \mathrm{C}$ for $15 \mathrm{~s}$. The expression levels of MKK genes were calculated according to Livak and Schmittgen ${ }^{56}$.

Yeast Two-hybrid Assays. Putative interactions between MKKs and MAPKs were examined using the matchmaker Gold Yeast Two-Hybrid system according to the manufacturer's instructions (Clontech, Mountain View, CA, USA). The coding sequences of MKKs and MAPKs were amplified using gene-specific primers from G. hirsutum acc. TM-1 and cloned into pGBKT7 and pGADT7 vectors (Supplementary Dataset 6). Eight MKK proteins were transformed into $\mathrm{Y} 2 \mathrm{H}$ Gold yeast strains and the auto-activation activity was tested. Aureobasidin A (ABA) was used to inhibit the auto-activation ${ }^{57}$. Individual MKK-MAPK pairs were co-transformed into yeast cells, and the resulting colonies were tested on selective medium (SD/-Trp/-Leu and SD/-Trp/-Leu/ABA), then on quadruple dropout medium (SD/-Ade/-His/-Leu/-Trp) and quadruple dropout medium supplemented with X- $\alpha-\mathrm{Gal}$ and Aureobasidin A for 7 days at $30^{\circ} \mathrm{C}$, only yeast colonies with interactions between MKKs and MAPKs were able to grow on the selection media. Co-transformation of pGBKT7-53, and pGBKT7-Lam and pGADT7-rec, were as positive and negative controls, respectively.

Statistical Analysis. All experiments were repeated independently at least three times. Data obtained were subjected to statistical analysis using student's t-tests and probability values of $\mathrm{P}<0.05$ were considered as significant between the different treatments.

\section{References}

1. Sinha, A. K., Jaggi, M., Raghuram, B. \& Tuteja, N. Mitogen-activated protein kinase signaling in plants under abiotic stress. Plant Signal. Behav. 6, 196-203 (2011).

2. Hamel, L. P. et al. Ancient signals: comparative genomics of plant MAPK and MAPKK gene families. Trends Plant Sci. 11, 192-198 (2006).

3. Jonak, C. Complexity, Cross talk and integration of plant MAP kinase signalling. Curr. Opin. Plant Biol. 5, 415-424 (2002).

4. Colcombet, J. \& Hirt, H. Arabidopsis MAPKs: a complex signalling network involved in multiple biological processes. Biochem. J. 413, 217-226 (2008).

5. Ichimura, K. et al. Mitogen-activated protein kinase cascades in plants: a new nomenclature. Trends Plant Sci. 7, 301-308 (2002).

6. Singh, R. et al. Rice mitogen-activated protein kinase interactome analysis using the yeast two-hybrid system. Plant Physiol. 160, 477-487 (2012)

7. Rao, K. P., Richa, T., Kumar, K., Raghuram, B. \& Sinha, A. K. In silico analysis reveals 75 members of mitogen-activated protein kinase kinase kinase gene family in rice. DNA Res. 17, 139-153 (2010).

8. Wu, J. et al. Genome-wide identification of MAPKK and MAPKKK gene families in tomato and transcriptional profiling analysis during development and stress response. PLoS One 9, e103032 (2014).

9. Kong, F. et al. Genome-wide analysis of the mitogen-activated protein kinase gene family in Solanum lycopersicum. Gene 499, 108-120 (2012)

10. Kong, X. et al. Identification of mitogen-activated protein kinase kinase gene family and MKK-MAPK interaction network in maize. Biochem. Biophys. Res. Commun. 441, 964-969 (2013).

11. Kong, X. et al. Genome-wide identification and analysis of expression profiles of maize mitogen-activated protein kinase kinase kinase. PLoS One 8, e57714 (2013).

12. Wang, J. et al. Genome-wide identification of MAPK, MAPKK, and MAPKKK gene families and transcriptional profiling analysis during development and stress response in cucumber. BMC Genomics 16, 386 (2015).

13. Jiang, M. et al. Genome-wide exploration of the molecular evolution and regulatory network of mitogen-activated protein kinase cascades upon multiple stresses in Brachypodium distachyon. BMC Genomics 16, 228 (2015).

14. Nakagami, H., Soukupova, H., Schikora, A., Zarsky, V. \& Hirt, H. A Mitogen-activated protein kinase kinase kinase mediates reactive oxygen species homeostasis in Arabidopsis. J. Biol. Chem. 281, 38697-38704 (2006).

15. Petersen, M. et al. Arabidopsis MAP Kinase 4 Negatively Regulates Systemic Acquired Resistance. Cell 103, 10 (2000).

16. Teige, M. et al. The MKK2 pathway mediates cold and salt stress signaling in Arabidopsis. Mol. Cell 15, 141-152 (2004). 
17. Gao, M. et al. MEKK1, MKK1/MKK2 and MPK4 function together in a mitogen-activated protein kinase cascade to regulate innate immunity in plants. Cell Res. 18, 1190-1198 (2008).

18. Furuya, T., Matsuoka, D. \& Nanmori, T. Membrane rigidification functions upstream of the MEKK1-MKK2-MPK4 cascade during cold acclimation in Arabidopsis thaliana. FEBS Lett. 588, 2025-2030 (2014).

19. Qiu, J. L. et al. Arabidopsis mitogen-activated protein kinase kinases MKK1 and MKK2 have overlapping functions in defense signaling mediated by MEKK1, MPK4, and MKS1. Plant Physiol. 148, 212-222 (2008).

20. Takahashi, F. et al. The mitogen-activated protein kinase cascade MKK3-MPK6 is an important part of the jasmonate signal transduction pathway in Arabidopsis. Plant Cell 19, 805-818 (2007).

21. Yue, H., Li, Z. \& Xing, D. Roles of Arabidopsis bax inhibitor-1 in delaying methyl jasmonate-induced leaf senescence. Plant Signal. Behav. 7, 1488-1489 (2012).

22. Danquah, A. et al. Identification and characterization of an ABA-activated MAP kinase cascade in Arabidopsis thaliana. Plant J. 82, 232-244 (2015)

23. Asai, T. et al. MAP kinase signalling cascade in Arabidopsis innate immunity. Nature 415, 977-983 (2002).

24. Galletti, R., Ferrari, S. \& De Lorenzo, G. Arabidopsis MPK3 and MPK6 play different roles in basal and oligogalacturonide- or flagellin-induced resistance against Botrytis cinerea. Plant Physiol. 157, 804-814 (2011).

25. Wang, H., Ngwenyama, N., Liu, Y., Walker, J. C. \& Zhang, S. Stomatal development and patterning are regulated by environmentally responsive mitogen-activated protein kinases in Arabidopsis. Plant Cell 19, 63-73 (2007).

26. Gudesblat, G. E., Iusem, N. D. \& Morris, P. C. Guard cell-specific inhibition of Arabidopsis MPK3 expression causes abnormal stomatal responses to abscisic acid and hydrogen peroxide. New Phytol. 173, 713-721 (2007).

27. Zeng, Q., Chen, J. G. \& Ellis, B. E. AtMPK4 is required for male-specific meiotic cytokinesis in Arabidopsis. Plant J. 67, 895-906 (2011).

28. Zhou, C., Cai, Z., Guo, Y. \& Gan, S. An Arabidopsis mitogen-activated protein kinase cascade, MKK9-MPK6, plays a role in leaf senescence. Plant Physiol. 150, 167-177 (2009).

29. Xu, J. et al. Activation of MAPK kinase 9 induces ethylene and camalexin biosynthesis and enhances sensitivity to salt stress in Arabidopsis. J. Biol. Chem. 283, 26996-27006 (2008).

30. Lu, W., Chu, X., Li, Y., Wang, C. \& Guo, X. Cotton GhMKK1 induces the tolerance of salt and drought stress, and mediates defence responses to pathogen infection in transgenic Nicotiana benthamiana. PLoS One 8, e68503 (2013).

31. Gao, X. et al. Silencing GhNDR1 and GhMKK2 compromises cotton resistance to Verticillium wilt. Plant J. 66, 293-305 (2011).

32. Li, Y. et al. Overexpression of cotton GhMKK4 enhances disease susceptibility and affects abscisic acid, gibberellin and hydrogen peroxide signalling in transgenic Nicotiana benthamiana. Mol. Plant Pathol. 15, 94-108 (2014).

33. Zhang, L. et al. Cotton GhMKK5 affects disease resistance, induces HR-like cell death, and reduces the tolerance to salt and drought stress in transgenic Nicotiana benthamiana. J. Exp. Bot. 63, 3935-3951 (2012).

34. Erik, A. \& Brian, E. Convergence and specificity in the Arabidopsis MAPK nexus. Trends Plant Sci. 15,106-113 (2010).

35. Yin, Z. et al. The MAPKKK gene family in Gossypium raimondii: genome-wide identification, classification and expression analysis. Int. J. Mol. Sci. 14, 18740-18757 (2013).

36. Zhang, X., Wang, L., Xu, X., Cai, C. \& Guo, W. Genome-wide identification of mitogen-activated protein kinase gene family in Gossypium raimondii and the function of their corresponding orthologs in tetraploid cultivated cotton. BMC Plant Biol. 14, 345 (2014).

37. Paterson, A. H. et al. Repeated polyploidization of Gossypium genomes and the evolution of spinnable cotton fibres. Nature 492, 423-427 (2012).

38. Zhang, T. et al. Sequencing of allotetraploid cotton (Gossypium hirsutum L. acc. TM-1) provides a resource for fibre improvement. Nat. Biotech. 33, 531-537 (2015).

39. Liu, X. et al. Gossypium barbadense genome sequence provides insight into the evolution of extra-long staple fiber and specialized metabolites. Sci. Rep. 5, 14139 (2015).

40. Li, F. et al. Genome sequence of the cultivated cotton Gossypium arboreum. Nat. Genet. 46, 567-572 (2014).

41. Wankhede, D. P., Misra, M., Singh, P. \& Sinha, A. K. Rice mitogen activated protein kinase kinase and mitogen activated protein kinase interaction network revealed by in-silico docking and yeast two-hybrid approaches. PLoS One 8, e65011 (2013).

42. Samajova, O., Plihal, O., Al-Yousif, M., Hirt, H. \& Samaj, J. Improvement of stress tolerance in plants by genetic manipulation of mitogen-activated protein kinases. Biotechnol. Adv. 31, 118-128 (2013).

43. Rodriguez, M. C., Petersen, M. \& Mundy, J. Mitogen-activated protein kinase signaling in plants. Annu. Rev. Plant Biol. 61, 621-649 (2010).

44. Lampard, G. R., Wengier, D. L. \& Bergmann, D. C. Manipulation of mitogen-activated protein kinase kinase signaling in the Arabidopsis stomatal lineage reveals motifs that contribute to protein localization and signaling specificity. Plant Cell 26, 3358-3371 (2014).

45. Beck, M., Komis, G., Muller, J., Menzel, D. \& Samaj, J. Arabidopsis homologs of nucleus- and phragmoplast-localized kinase 2 and 3 and mitogen-activated protein kinase 4 are essential for microtubule organization. Plant Cell 22, 755-771 (2010).

46. Dai, Y. et al. Increased expression of MAP KINASE KINASE7 causes deficiency in polar auxin transport and leads to plant architectural abnormality in Arabidopsis. Plant Cell 18, 308-320 (2006).

47. Duan, P. et al. SMALL GRAIN 1, which encodes a mitogen-activated protein kinase kinase 4 , influences grain size in rice. Plant $J$. 77, 547-557 (2014)

48. Takahashi, F., Mizoguchi, T., Yoshida, R., Ichimura, K. \& Shinozaki, K. Calmodulin-dependent activation of MAP kinase for ROS homeostasis in Arabidopsis. Mol. Cell 41, 649-660 (2011).

49. Doczi, R. et al. The Arabidopsis mitogen-activated protein kinase kinase MKK3 is upstream of group C mitogen-activated protein kinases and participates in pathogen signaling. Plant Cell 19, 3266-3279 (2007).

50. Zhang, X. et al. Overexpression of Arabidopsis MAP kinase kinase 7 leads to activation of plant basal and systemic acquired resistance. Plant J. 52, 1066-1079 (2007).

51. Lee, J. S., Huh, K. W., Bhargava, A. \& Ellis, B. E. Comprehensive analysis of protein-protein interactions between Arabidopsis MAPKs and MAPK kinases helps define potential MAPK signalling modules. Plant Signal. Behav. 3, 1037-1041 (2008).

52. Singh, R. \& Jwa, N. S. The rice MAPKK-MAPK interactome: the biological significance of MAPK components in hormone signal transduction. Plant Cell Rep. 32, 923-931 (2013).

53. Liang, W. et al. Identification and analysis of MKK and MPK gene families in canola (Brassica napus L.). BMC Genomics 14, 392 (2013).

54. Song, Q. et al. Characterization, expression patterns and functional analysis of the MAPK and MAPKK genes in watermelon (Citrullus lanatus). BMC Plant Biol. 15, 298 (2015).

55. Shi, J., An, H. L., Zhang, L., Gao, Z. \& Guo, X. Q. GhMPK7, a novel multiple stress-responsive cotton group C MAPK gene, has a role in broad spectrum disease resistance and plant development. Plant Mol. Biol. 74, 1-17 (2010).

56. Livak, K. J. \& Schmittgen, T. D. Analysis of relative gene expression data using real-time quantitative PCR and the $2^{-\Delta \Delta \mathrm{CT}}$ method. Methods 25, 402-408 (2001).

57. Serebriiskii, I., Estojak, J., Berman, M. \& Golemis, E. A. Approaches to detecting false positives in yeast two-hybrid systems. Biotechniques 28, 332-336 (2000). 


\section{Acknowledgements}

This program was financially supported in part by National Science Foundation in China (31171590), Key R\&D program in Jiangsu Province (BE2015360), six talent peaks project in Jiangsu province (2015-NY-002), the project for Student Research Training in Jiangsu province (201510307003Y), the Priority Academic Program Development of Jiangsu Higher Education Institutions (010-809001), and JCIC-MCP project (No. 10).

\section{Author Contributions}

Experiments were designed by W.G. Experiments were performed by X.Z., X.X., Y.Y., C.C., J.W. and C.C. X.Z. and W.G. drafted the manuscript, W.G. and X.Z. revised the manuscript. All authors read and approved the final manuscript.

\section{Additional Information}

Supplementary information accompanies this paper at http://www.nature.com/srep

Competing financial interests: The authors declare no competing financial interests.

How to cite this article: Zhang, X. et al. Integration analysis of MKK and MAPK family members highlights potential MAPK signaling modules in cotton. Sci. Rep. 6, 29781; doi: 10.1038/srep29781 (2016).

(c) (i) This work is licensed under a Creative Commons Attribution 4.0 International License. The images or other third party material in this article are included in the article's Creative Commons license, unless indicated otherwise in the credit line; if the material is not included under the Creative Commons license, users will need to obtain permission from the license holder to reproduce the material. To view a copy of this license, visit http://creativecommons.org/licenses/by/4.0/ 